\section{CONFIANZA EN LA MARCA PERIODÍSTICA Y ADOPCIÓN DE LA PRENSA ON-LINE}

\section{Ivan Lacasa Mas}

\section{Profesor adjunto}

Facultad de Ciencias de la Comunicación. Universitat Internacional de Catalunya. Immaculada 22, 08017, Barcelona (España) - Email: lacasa@cir.uic.es

\section{María Victoria Mas}

\section{Profesora ayudante}

Facultad de Ciencias de la Comunicación. Universitat Internacional de Catalunya. Immaculada 22, 08017, Barcelona (España) - Email: mvictoria@cir.uic.es

\section{Mariano Fernández Diez}

\section{Marketing Manager}

Lavanguardia.com - Email: mfernandez@lavanguardia.es

\section{Resumen}

Las cabeceras de prensa tradicionales se encuentran en la fase inicial de un proceso convergente que les conduce hacia una distribución multiplataforma de contenidos. Tras el análisis de los resultados de una encuesta realizada a más de 3.500 lectores de LaVanguardia que habitualmente leen tanto la edición impresa como la digital, concluimos que las motivaciones que hoy les mueven a ese consumo en el que los dos soportes no compiten están más relacionadas con la confianza en la

\section{Palabras clave}

Prensa on-line, soporte impreso, usuarios, marca periodística, gratificaciones, multiplataforma

Key Words

On-line newspaper, printed newspaper, users, journalistic brand, gratifications, multiplatform

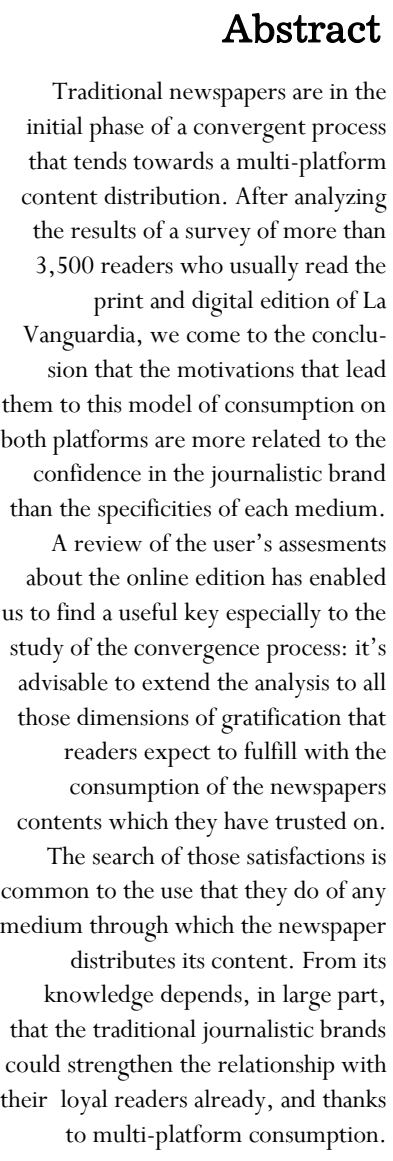

\section{Abstract}

Traditional newspapers are in the initial phase of a convergent process that tends towards a multi-platform content distribution. After analyzing the results of a survey of more than print and digital edition of $\mathrm{La}$ anguardia, we come to the concluoth platforms are more related to the confidence in the journalistic brand an the specificities of each medium. A review of the user's assesments about the online edition has enabled us to find a useful key especially to the study of the convergence process: it's advisable to extend the analysis to all ose dimensions of gratification that readers expect to fulfill with the

The search of those satisfactions is common to the use that they do of any medium through which the newspaper distributes its content. From its knowledge depends, in large part, that the traditional journalistic brands could strengthen the relationship with to multi-platform consumption. 
marca periodística, que con las especificidades de cada uno de dichos soportes. El examen de las valoraciones que esos usuarios hacen de la edición on-line nos ha permitido dar con una clave especialmente útil para el estudio del proceso convergente: conviene que el análisis de los usuarios de la prensa on-line se amplíe e incluya todas aquellas gratificaciones que los lectores esperan satisfacer no solo a través del uso de cada soporte, sino también mediante la relación con la marca periodística en la que han depositado su confianza. La búsqueda de esas gratificaciones es la que hoy mueve a un grupo muy significativo de lectores a usar los dos soportes a través de los cuales las cabeceras tradicionales distribuyen sus contenidos. Del conocimiento de las gratificaciones relacionadas con la marca periodística depende, en gran parte, el que las cabeceras puedan reforzar la relación con sus lectores gracias a la distribución multiplataforma ${ }^{\mathrm{i}}$.

\section{Introducción}

Los diarios on-line empiezan ya a consolidarse dentro de las empresas periodísticas tradicionales (Masip et al., 2010). Estas empresas se encuentran tan solo en la fase inicial de un proceso de convergencia en el que, entre otras muchas cosas, queda precisamente por definir qué relación está llamada a establecerse entre las dos ediciones de cada cabecera de prensa tradicional y, en consecuencia, cómo debería llevarse a cabo la coordinación entre el soporte online y el soporte impreso (Díaz-Noci, 2010; López y Pereira, 2010) ${ }^{\mathrm{ii}}$. Hoy permanece abierto el interrogante acerca de la dirección hacia la que está llamada a evolucionar la prensa: hacia una distribución en ambos soportes o hacia una distribución exclusivamente digital. Hay quien afirma que todos los contenidos que hoy producen las cabeceras periodísticas podrían migrar a Internet sin demasiadas dificultades por parte del público, puesto que los ya quince años de experiencia en prensa on-line y la mayor facilidad de acceso derivada de la adopción de los dispositivos móviles han conseguido que gran parte de los usuarios estén preparados para usar de forma generalizada el periódico digital (Smith, 2011) (ii $^{\mathrm{ii}}$. Será útil, por tanto, estudiar desde la perspectiva del usuario esta cuestión que atañe a las cabeceras periodísticas tradicionales.

$\mathrm{Si}$ atendemos a los datos de las oleadas realizadas entre los años 2008 y 2010 por el Estudio General de Medios (EGM) sobre la penetración de las principales cabeceras de prensa españolas ${ }^{\text {iv }}$ vemos que el número de personas que consultan exclusivamente la versión digital crece de forma ininterrumpida (vid. Cuadro 1$)^{\mathrm{v}}$. 
Cuadro $n^{\circ} 1$. Variación de la lectura de cada soporte en las principales cabeceras de prensa españolas

\begin{tabular}{|c|c|c|}
\hline Cabecera & Edición & $\begin{array}{c}\text { Variación de la } \\
\text { penetración desde } \\
2008 \text { (EGM) }\end{array}$ \\
\hline \multirow{3}{*}{ El País } & impresa & $-14,11 \%$ \\
\hline & digital & $41,63 \%$ \\
\hline & ambas & $3,90 \%$ \\
\hline \multirow{3}{*}{ El Periódico } & impresa & $-4,10 \%$ \\
\hline & digital & $72.31 \%$ \\
\hline & ambas & $37,20 \%$ \\
\hline \multirow{3}{*}{$\mathbf{A B C}$} & impresa & $6,97 \%$ \\
\hline & digital & $128,76 \%$ \\
\hline & ambas & $113,64 \%$ \\
\hline \multirow{3}{*}{ La Vanguardia } & impresa & $2,07 \%$ \\
\hline & digital & $61,74 \%$ \\
\hline & ambas & $48,33 \%$ \\
\hline
\end{tabular}

Fuente: $E G M$

Esos mismos datos señalan que, si bien el número de personas que leen ambas ediciones también aumenta, la audiencia continúa altamente interesada en la información de actualidad del soporte papel, pues los lectores que consumen exclusivamente la edición impresa siguen constituyendo una mayoría claramente dominante (vid. Gráficos. 1-4) ${ }^{\mathrm{vi}}$.

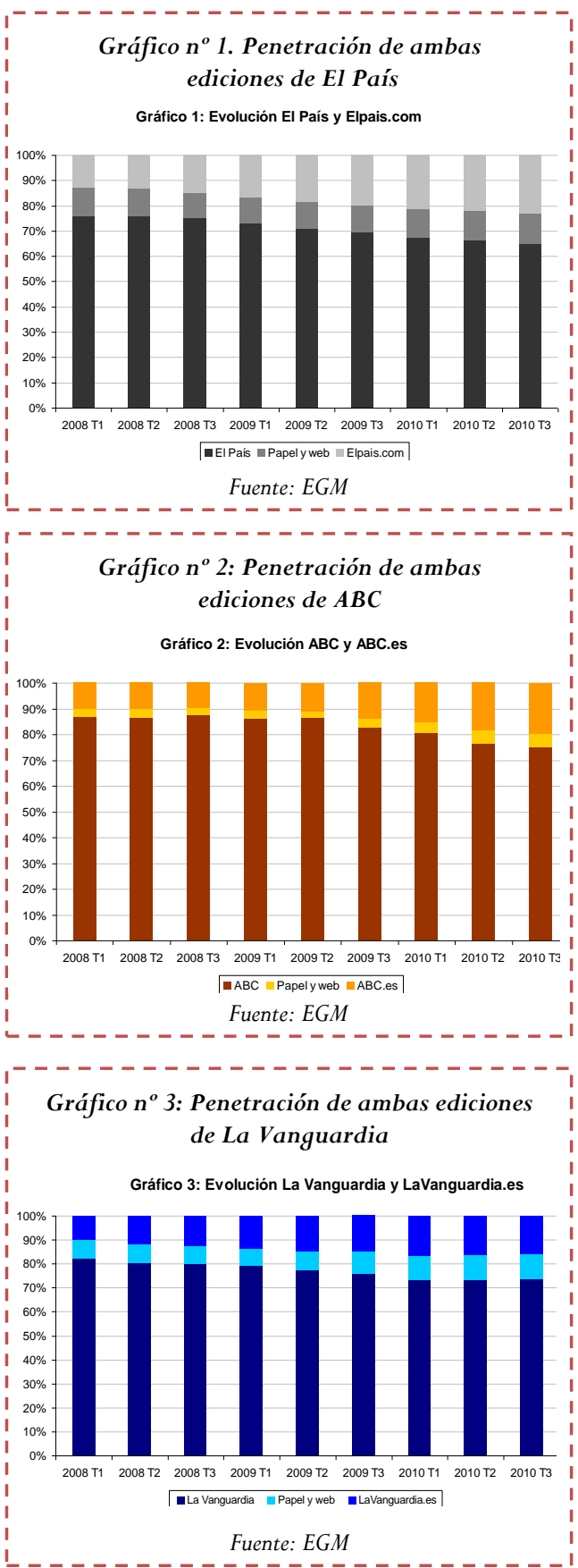




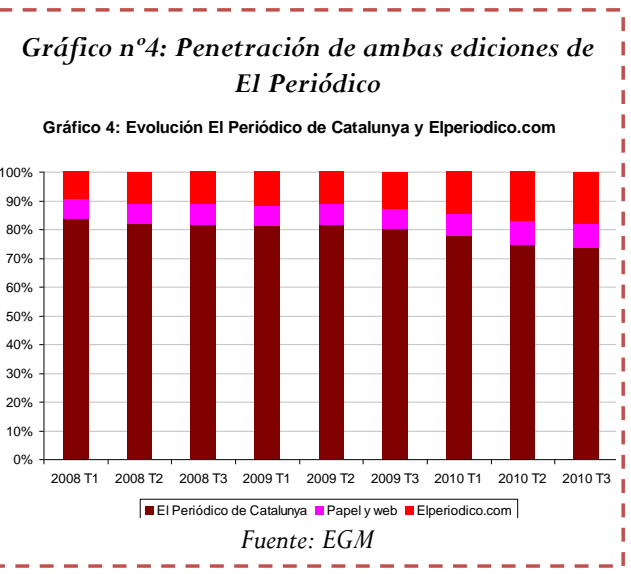

El mercado actual de las cabeceras de prensa parece encontrarse, por tanto, en un periodo de transición en el que cada marca periodística posee tres tipos de consumidores: los lectores de la edición impresa, los lectores "híbridos" y los lectores de la edición on-line (Chyi et. al., 2010). Siendo el primer grupo de lectores el más numeroso, podemos afirmar que la edición impresa es, al menos desde el punto de vista de la demanda, un producto hoy todavía plenamente competitivo (Chyi y Huang, 2011; Valdettaro, 2009; Picard 2003). De todas maneras, visto el crecimiento de los otros dos grupos, cabe reformular el interrogante abierto más arriba y preguntarse hacia dónde está llamada a evolucionar la demanda de los contenidos que las empresas de prensa tradicional producen: ¿hacia un consumo en ambos soportes o hacia uno exclusivamente digital?

\section{Objetivos}

En el presente artículo analizaremos las valoraciones que hacen de la edición impresa y digital de una cabecera de prensa de referencia como es La Vanguardia aquellas personas que habitualmente leen tanto una edición como la otra. Analizar cuáles pueden estar siendo los motivos que les mue- ven a adoptar el uso de la edición on-line, sin por ello dejar de consumir la edición impresa, podría ayudarnos a determinar hasta qué punto el consumo en ambos soportes es el que está llamado a generalizarse entre los lectores de la prensa impresa.

\section{Metodología}

Según los datos de la última oleada del EGM de 2010, del total de usuarios de la LaVanguardia.com un 40\% combina su lectura con la de la edición impresa. Para estudiar a esa población, entre los meses de octubre a diciembre de 2010 hemos realizamos una extensa encuesta a 3.593 lectores registrados ${ }^{\text {vii }}$ en LaVanguardia.com ${ }^{\text {viii }}$, unos usuarios que mantienen un vínculo con ambas ediciones de ese diario. 
Si tenemos en cuenta que el 50,97\% de los encuestados hace más de dos años que conoce LaVanguardia.com y que en el $40,15 \%$ de los casos esa antigüedad asciende hasta más allá de los 5 años, bien podemos afirmar que sus patrones de consumo se asientan sobre unos hábitos desarrollados durante un periodo de tiempo considerable, lo cual nos da a entender que los lectores encuestados tienen una relación ya madura con la edición digital, así como con su cabecera periodística. A fin de ajustarnos a los objetivos del presente artículo, limitaremos los datos analizados a las respuestas de aquellos lectores que consumen a diario o muy frecuentemente (2-5 veces a la semana) la edición on-line. Son más de un $70 \%$ de los encuestados. Además, de entre estos lectores habituales de la edición online hemos escogido las respuestas de aquellos que también leen la edición impresa, con dos tipos de frecuencia: habitual (a diario o 2-5 veces a la semana) y mínima (una vez a la semana) (vid. Cuadro 2).
Cuadro $n^{\circ}$ 2. Muestras escogidas para el análisis de los datos

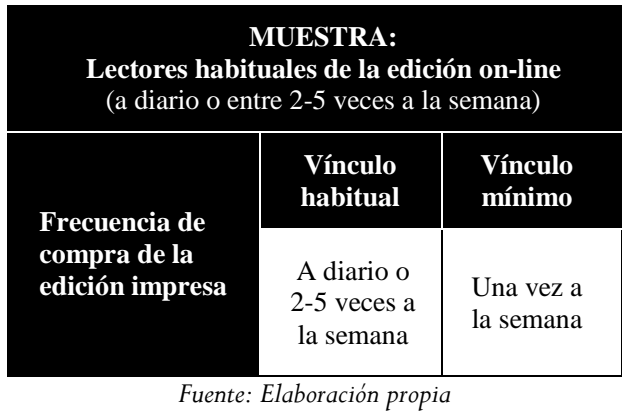

El análisis de las respuestas de estos dos tipos de perfiles de usuarios ha ido dirigido a determinar si sus expectativas con respecto a la edición on-line están relacionadas con las expectativas que poseen de la edición impresa y hasta qué punto pueden estar influidas por la tradición de uso que tienen de este soporte. La división por frecuencias de lectura de la prensa impresa permitirá discernir si el uso más o menos intenso del soporte tradicional influye en las expectativas de uso y relación con la edición on-line.

\section{Marco conceptual: prensa impresa y prensa on-line, ¿sustitutivas o complementarias?}

Con frecuencia, el estudio de la relación que puede estar estableciéndose entre las ediciones impresas y digitales de las cabeceras de prensa tradicional ha heredado sin matices suficientes la perspectiva general, comúnmente asumida por la investigación, acerca del efecto de desplazamiento que Internet puede ejercer sobre los medios tradicionales y, más en concreto, sobre el periódico impreso (Mo Park, 2001; Van der Wuurf, 2005; Filistruchi, 2005; George, 2006; Van der Wuurf et. al., 2008; Westlund y Färlich, 2011) ${ }^{\text {ix }}$. Por ello, tal estudio ha partido en muchas ocasiones de la premisa de que las ediciones digitales compiten directamente con sus ediciones 
impresas y pueden desplazarlas hasta llegar a sustituirlas en un futuro próximo (Chyi y Lasorsa, 2002 y 2006; de Waal et. al. 2005; Gentzow, 2006; Flavián y Gurrea, 2007; Chyi y Huang, 2011).

Dentro de la perspectiva usuario-céntrica, que aborda esa cuestión de la competencia entre prensa impresa y prensa on-line a través del estudio de la demanda y las preferencias de los usuarios, una de las principales líneas de investigación es la que analiza la penetración de cada una de ellas, a fin de determinar si el aumento del consumo de prensa on-line implica una sustitución del consumo de prensa impresa o si, por el contrario, parece convivir con él, en cuyo caso podría presumirse que entre ambos tipos de prensa puede estar estableciéndose una relación de complementariedad (Chyi y Lasorsa, 2002; de Waal et. al., 2005; Filistruchi, 2005; Chyi y Huang, 2011). Autores como de Waal, Schönbach y Lauf afirman, por ejemplo, que el consumo de diarios on-line está negativamente relacionado con el consumo de prensa impresa, pero solo en el caso de los lectores más jóvenes, los cuales representan una pequeña porción del público lector de periódicos (2005: 60). Por ello concluyen que es probable que entre prensa impresa y online se establezca una relación complementaria, puesto que no parece que el creciente consumo de los diarios digitales vaya a convertir en superfluo al conjunto de funciones informativas de los diarios impresos (de Waal et al., 2005: 60). En esa misma línea, cuando la disyuntiva sustitucióncomplementariedad se ha trasladado al estudio del caso de las ediciones on-line e impresa de una misma cabecera, más específico que el de la relación entre papel e Internet y que el de la relación entre prensa impresa y on-line en general, a la vista de los datos de penetración la mayoría de estudios han defendido que hoy el soporte impreso sigue siendo altamente competitivo, al menos en lo que al número de lectores se refiere (Chyi y Lasorsa 2002; Gentzow, 2006; Flavián y Gurrea, 2007; Chyi y Huang, 2011). Algunos de estos autores sostienen, incluso, que entre los usuarios de las ediciones on-line no ha disminuido la penetración de las ediciones impresas y que parte importante de los lectores de una misma cabecera hace un consumo simultáneo de ambas ediciones (Chyi y Lasorsa, 2002; Chyi y Huang, 2011).

A raíz de lo visto hasta aquí, podemos afirmar que, si bien la literatura que ha examinado el caso específico de las ediciones de prensa de una misma marca periodística es escasa, la mayoría de las investigaciones que estudian esta cuestión centrándose en la demanda o en las preferencias del usuario concluyen unánimemente que es posible pensar en una convivencia entre ambas ediciones y que, por tanto, entre ellas podría estar estableciéndose una relación no de competencia, sino de complementariedad (de Waal et al., 2005; Chyi y Lasorsa, 2002; Chyi, 2006; Flavián y Gurrea, 2007; Chyi y Huang, 
2011; Westlund y Färlich, 2011). Sobre todo si resultara posible afirmar que existe un consumo simultáneo de ambas ediciones de prensa, también podría pensarse que los lectores están utilizando cada una de ellas de modo distinto o con fines diversos, lo cual, a su vez, nos permitiría pronunciarnos con más fundamento a favor de la tesis que sostiene la existencia de una relación de complementariedad entre las dos ediciones. No obstante, a fin de comprender en qué consiste dicha relación complementaria, varias de las investigaciones citadas remarcan la necesidad de profundizar no solo en si los lectores usan las diferentes ediciones de cada cabecera de prensa, sino en por qué y cómo las usan (Chyi y Lasorsa, 2002; de Waal et al., 2005).

Una línea de investigación que resulta útil para explicar en qué parece consistir esa complementariedad que los lectores pueden estar estableciendo entre las ediciones de prensa de una misma cabecera es la que parte del enfoque de usos y gratificaciones ${ }^{\mathrm{x}}$. Esta perspectiva se ha adoptado no pocas veces a la hora de estudiar la adopción de los nuevos medios, de ahí que desde ella se haya abordado también la cuestión sobre la competencia entre la red y los medios tradicionales (Ruggiero, 2000). Son estudios que tratan de discernir los motivos que llevan a los lectores a escoger Internet como fuente de información; buscan con ello poder compararlos con los que mueven a consumir los medios tradicionales, y en concreto, el periódico im- preso, a fin de determinar si Internet puede dar una mayor o mejor respuesta a las necesidades que los medios tradicionales satisfacen y, por ello, acabar sustituyéndolos (Dimmick, 2003; Dimmick et. al. 2004; Lin et. al., 2005; Chung y Yun Yoo, 2006; Flavián y Gurrea, 2007; Chung, 2008). Cabe destacar que también la mayoría de estos estudios se han realizado desde una perspectiva muy amplia que analiza la competencia entre Internet y los periódicos tradicionales de manera global, es decir, entendiéndolos como dos soportes que compiten, pero sin tener en cuenta los medios informativos concretos que a través de ellos difunden sus contenidos (Chiy y Huang, 2011: 93). A esa perspectiva pertenecen los estudios que combinan el enfoque de usos y gratificaciones con la «teoría del nicho» ${ }^{x i}$, los cuales subrayan la superioridad del soporte digital y afirman que, en general, produce un desplazamiento del uso de los medios tradicionales que hace pensar en una futura sustitución (Dimmick et. al., 2004). Sin embargo, según esos mismo estudios, tal superioridad no proviene de la capacidad del soporte digital de satisfacer las tres necesidades clásicas asociadas a los medios de comunicación: información, entretenimiento o interacción social (Dimmick et. al., 2004). Lo que esos autores subrayan no es el hecho de que Internet satisface más necesidades que los otros medios, sino que ofrece mayor diversidad de oportunidades de gratificación a la hora de hacerlo: la flexibi- 
lidad para el consumo de los contenidos informativos que presenta el soporte digital hace que el usuario posea más opciones para escoger el tipo de información que consume, el lugar y el momento en el que lo hace (Dimmick, 2003: 103; Dimmick et. al., 2004).

No obstante, cuando algunas investigaciones han trasladado el punto de mira desde las ventajas que ofrecen las características específicas del soporte o canal mediático hasta las gratificaciones que los lectores dicen obtener ${ }^{x i i}$ en el uso específico de determinados medios informativos on-line, y las han comparado con aquellas que afirman recibir al usar el soporte impreso, han concluido que las gratificaciones a las que conduce la edición on-line no hacen que los lectores la perciban como superior a la edición impresa, menos aún como sustitutoria (Flavián y Gurrea, 2007: 798). Mientras el soporte digital presenta más ventajas que el soporte papel para la satisfacción de necesidades genéricas como el consumo de información específica o como la búsqueda de noticias actualizadas, el periódico impreso continúa siendo el preferido por los lectores para satisfacer otras necesidades, algunas de ellas más relacionadas con los momentos de ocio ${ }^{\text {xiii }}$ (Flavián y Gurrea, 2007; Chung, 2008). Estos resultados contrastan con las conclusiones de aquellos estudios que subrayan la superioridad del soporte digital: las oportunidades de gratificación que aporta no son percibidas por los lectores como un motivo para dejar de leer o de valorar la edición impresa, ya que asocian otro tipo de gratificaciones al papel como soporte. De ahí que podamos considerar que son precisamente las características distintivas de cada soporte las que causan que estos no sean percibidos como sustitutorios, sino como complementarios, a la hora de satisfacer unas mismas necesidades que los lectores buscan en la lectura de prensa en general (Flavián y Gurrea, 2007: 798).

A la luz de todo lo visto en el presente apartado, cabe preguntarse hasta qué punto aquellos lectores de la edición impresa que, sin dejar de leerla, adoptan el uso de la edición on-line, pueden estar valorando las especificidades del soporte digital y considerándolo superior para el consumo de los contenidos de su cabecera de prensa. Solo así será posible determinar algunos de los principales factores subjetivos que rigen los patrones de consumo de los lectores que hoy acceden a los contenidos de prensa en ambas plataformas y determinar hasta qué punto este tipo de consumo en ambos soportes está llamado a extenderse. 


\section{Discusión de los datos: estudio de usuarios de LaVanguardia.com}

Mediante el análisis de una selección de preguntas de la extensa encuesta que hemos realizado a aquellos lectores de La Vanguardia.com que leen también La Vanguardia en formato impreso, vamos a tratar de profundizar en las percepciones que esas personas tienen de una y otra edición de esa misma cabecera de prensa ${ }^{\text {xiv }}$. Será una manera de comprobar hasta qué punto los lectores que adoptan el uso del soporte online para el consumo de la edición digital lo hacen motivados por las gratificaciones diferenciales percibidas que este soporte les proporciona.

\subsection{Valoraciones de los lectores sobre la edición impresa y la edición on-line}

Las afirmaciones referidas a la versión impresa con las que se identifican tanto los lectores que la compran frecuentemente —vínculo habitual_ como los lectores que lo hacen solo una vez a la semana vínculo mínimo- son muy similares (vid. Cuadro 3).

\begin{abstract}
Cuadro $n^{o}$ 3. Valoraciones de los lectores sobre la edición impresa
\end{abstract}

\begin{tabular}{|c|c|c|}
\hline & $\begin{array}{r}\text { vínculo } \\
\text { habitual }\end{array}$ & $\begin{array}{l}\text { vínculo } \\
\text { mínimo }\end{array}$ \\
\hline $\begin{array}{l}\text { Me gustan los reportajes a } \\
\text { fondo que analizan un } \\
\text { tema en concreto }\end{array}$ & 4,05 & 4,04 \\
\hline $\begin{array}{l}\text { Lo más importante en el } \\
\text { periódico es que sea actual }\end{array}$ & 4 & 4,31 \\
\hline $\begin{array}{l}\text { Compro La Vanguardia } \\
\text { porque es más objetiva que } \\
\text { otras cabeceras }\end{array}$ & 3,56 & 3,82 \\
\hline $\begin{array}{l}\text { Me identifico con la línea } \\
\text { editorial de La Vanguardia }\end{array}$ & 3,51 & 3,76 \\
\hline $\begin{array}{l}\text { El periódico es más objeti- } \\
\text { vo y veraz que otros me- } \\
\text { dios de información }\end{array}$ & 3,36 & 3,78 \\
\hline $\begin{array}{l}\text { Un periódico debe ser } \\
\text { entretenido y divertido }\end{array}$ & 3,25 & 3,49 \\
\hline $\begin{array}{l}\text { Normalmente me intereso } \\
\text { solo por algunas secciones } \\
\text { en concreto }\end{array}$ & 3,29 & 3,39 \\
\hline $\begin{array}{l}\text { Prefiero las noticias infor- } \\
\text { mativas antes que los artí- } \\
\text { culos de opinión }\end{array}$ & 3,12 & 3,13 \\
\hline $\begin{array}{l}\text { Lo que más me interesa } \\
\text { son las columnas de opi- } \\
\text { nión }\end{array}$ & 2,93 & 3,15 \\
\hline $\begin{array}{l}\text { Las noticias locales son } \\
\text { más importantes que las } \\
\text { noticias internacionales }\end{array}$ & 2,78 & 2,89 \\
\hline $\begin{array}{l}\text { Normalmente ojeo los } \\
\text { titulares pero no me paro a } \\
\text { leer a fondo las noticias }\end{array}$ & 2,63 & 2,84 \\
\hline $\begin{array}{l}\text { Compro el periódico cuando } \\
\text { me interesa un tema en } \\
\text { concreto }\end{array}$ & 2,05 & 1,68 \\
\hline $\begin{array}{l}\text { Solo compro el periódico } \\
\text { cuando viene acompañado } \\
\text { de un suplemento que me } \\
\text { gusta }\end{array}$ & 1,58 & 1,53 \\
\hline $\begin{array}{l}\text { Participo frecuentemente } \\
\text { enviando cartas al director }\end{array}$ & 1,39 & 1,43 \\
\hline
\end{tabular}

Fuente: Datos de la encuesta realizada por los autores a los usuarios de LaVanguardia.com 
Las que generan un mayor grado de acuerdo son "me gustan los reportajes a fondo que analizan un tema en concreto", "lo más importante en el periódico es que sea actual”, "compro La Vanguardia porque es más objetiva que otras cabeceras" y "me identifico con la línea editorial de La Vanguardia”.

El examen de estas valoraciones nos ayuda a trazar el perfil del periódico impreso que los lectores esperan encontrar. Para ellos un periódico es algo que tiene que ser "actual” y que es "más objetivo y veraz que otros medios de información". No es extraño, por tanto, que la afirmación con la que más se identifican sea la que valora la profundidad de los análisis. Tampoco lo es que, siendo lectores habituales de La Vanguardia, trasladen los valores que acabamos de señalar a la marca periodística y la definan como objetiva, tal y como puede verse en la tercera afirmación de la tabla.

Siendo las anteriores expectativas las más relevantes acerca de la edición impresa, se entiende que las afirmaciones referidas a la función de entretenimiento y a las columnas de opinión no ocupen un lugar preferente en la mente de los lectores ${ }^{\mathrm{xv}}$. Por otra parte, los lectores tampoco se identifican con aquellas afirmaciones que denotarían una lectura superficial del periódico, por ejemplo, ojear los titulares o no leer a fondo las noticias. Lo mismo sucede con aquellas afirmaciones relacionadas con motivos de compra del periódico que de- notan poca frecuencia o una baja valoración del producto periodístico (vid. Cuadro 3).

Con respecto a la versión digital (vid. Cuadro 4), la sentencia con la que se identifican más lectores es "aunque leo la versión digital, me gusta más el periódico de papel". Consideramos oportuno destacar que los encuestados que mantienen un vínculo mínimo con el diario impreso son los que manifiestan un mayor grado de acuerdo con esta afirmación, lo cual indica que leer con poca frecuencia la edición en papel no conlleva una menor valoración de este soporte, ni de los ideales que encarna. Además, al ser esta la única afirmación a la que ambos tipos de lectores se adhieren con claridad, cabe verla como una clave de interpretación para el resto de valoraciones, las cuales despiertan en ellos una adhesión débil, y están mucho más asociadas a funcionalidades o características específicas de la edición on-line.

\section{Cuadro no 4: Valoraciones de los lectores sobre la edición on-line}

\begin{tabular}{|l|c|c|}
\hline \multicolumn{2}{|c|}{$\begin{array}{c}\text { vínculo } \\
\text { habitual }\end{array}$} & $\begin{array}{c}\text { vínculo } \\
\text { mínimo }\end{array}$ \\
\hline $\begin{array}{l}\text { Aunque leo la versión digi- } \\
\text { tal me gusta más el periódi- } \\
\text { co de papel }\end{array}$ & 3,8 & 4,24 \\
\hline $\begin{array}{l}\text { La versión digital es de más } \\
\text { fácil consulta }\end{array}$ & 3,43 & 3,01 \\
\hline $\begin{array}{l}\text { Prefiero LaVanguar- } \\
\text { dia.com porque aporta } \\
\text { información más actuali- } \\
\text { zada que la versión en } \\
\text { papel }\end{array}$ & 3,23 & 3,06 \\
\hline $\begin{array}{l}\text { Leo la versión digital por- } \\
\text { que es gratis }\end{array}$ & 3,19 & 3,01 \\
\hline $\begin{array}{l}\text { Leo la versión digital } \\
\text { porque me aporta la mis- } \\
\text { ma información que el } \\
\text { papel de manera más fácil }\end{array}$ & 2,95 & 2,73 \\
\hline
\end{tabular}




\begin{tabular}{|l|c|c|}
\hline $\begin{array}{l}\text { Leo la versión digital por- } \\
\text { que puedo hacerlo mientras } \\
\text { trabajo }\end{array}$ & 3,17 & 2,71 \\
\hline $\begin{array}{l}\text { Leo tanto el papel como el } \\
\text { digital porque son cosas } \\
\text { distintas }\end{array}$ & 3,13 & 3,19 \\
\hline $\begin{array}{l}\text { La versión digital es más } \\
\text { entretenida que la impresa }\end{array}$ & 2,52 & 2,57 \\
\hline $\begin{array}{l}\text { Lo que más me gusta de la } \\
\text { versión digital es poder } \\
\text { opinar }\end{array}$ & 2,35 & 2,36 \\
\hline $\begin{array}{l}\text { La información del periódi- } \\
\text { co de papel me da más } \\
\text { confianza que la de la ver- } \\
\text { sión digital }\end{array}$ & 2,34 & 2,78 \\
\hline $\begin{array}{l}\text { La información de la ver- } \\
\text { sión digital es más objetiva }\end{array}$ & 2,07 & 2,11 \\
\hline
\end{tabular}

Fuente: Datos de la encuesta realizada por los autores a los usuarios de LaVanguardia.com

Las valoraciones que comparten los lectores con ambos tipos de vínculo y que destacan por encima de los 3 puntos son aquellas que tienen que ver con la accesibilidad, la inmediatez y la actualidad de la información: "la versión digital es de más fácil consulta”, "prefiero LaVanguardia.com porque aporta información más actualizada que la versión en papel" y "leo la versión digital porque es gratis". No obstante, el hecho de que estas afirmaciones no cuenten con un apoyo que resulte significativo induce a pensar que las características del soporte on-line de las que hablan no son esenciales para la elección y el uso de la edición digital. Ejemplo de ello es la valoración que recibe la afirmación "leo tanto el papel como el digital porque son cosas distintas", ya que con la puntuación que le otorgan, los lectores encuestados muestran no establecer una diferencia radical entre una y otra edición, lo cual, a su vez, explica que tampoco le concedan excesiva relevancia a aquellas funcionalidades técnicas del soporte on-line que lo distinguen del soporte papel.

Por su parte, las afirmaciones que constituyen comparaciones entre características del soporte tradicional y el digital revelan que ambos tipos de lectores valoran la edición on-line de forma muy similar a la edición impresa. Según los datos que mostraba el cuadro 3, comentado más arriba, la confianza y la objetividad, dos de las características primordiales que debe reunir un periódico, son atribuidas por los usuarios también a la edición on-line, en la medida en que, a su juicio, esta no las posee en un grado menor que la impresa (vid. cuadro 4). De hecho, podemos decir que juzgan de modo similar la edición on-line y la impresa, ya que no se identifican con la afirmación que concede más confiabilidad a la segunda y, además, otorgan a la edición online el mismo grado de objetividad que le otorgaban a la edición en papel.

Finalmente, las funciones relacionadas con el entretenimiento y la participación, que en la versión on-line, gracias a su dimensión multimedia e interactiva, podrían tener más peso en las expectativas de uso de los usuarios, reciben una valoración baja, igual que sucedía en el caso del periódico impreso. 


\section{Conclusiones}

No es mucha la bibliografía que ha estudiado el caso específico de la relación entre las ediciones on-line e impresa de una misma cabecera de prensa. La mayoría de investigaciones se han centrado en un objeto más amplio, a saber, la competencia entre los grandes medios tradicionales e Internet. Cuando algunos estudios se han limitado a la prensa y han estudiado la relación entre los periódicos impresos y on-line, lo han hecho heredando de esas investigaciones más generales la premisa de la competencia. No es de extrañar, por tanto, que su examen se haya detenido en desentrañar si la prensa on-line está llamada a sustituir a la impresa o, por el contrario, lo que va a hacer es complementarse con ella. Esta segunda hipótesis es la que validan los estudios que han analizado no tanto las características de los medios en sí, como el uso que de ellos hacen las personas: los medios que hoy conviven tienen que estar respondiendo de manera diversa a quienes se sirven de ellos para unos mismos fines. Al tratar de discernir qué gratificaciones específicas asocia el usuario a cada uno de los medios, los investigadores acaban hablando de aquellas directamente relacionadas con lo más específico de cada medio, el soporte mismo. Por ello, en estas páginas hemos tratado de analizar la percepción que tienen de la edición digital y de la impresa aquellos usuarios que hoy leen tanto una como otra, para ver hasta qué punto, al adoptar el uso del soporte on-line, lo que valoran son sus especificidades y, por tanto, lo que esperan es poder hacer un uso complementario de ambas ediciones. Gracias a la encuesta realizada a los usuarios de una de las cabeceras de prensa de referencia en España, hemos constatado que su percepción de la edición digital no está relacionada principalmente con las especificidades que el soporte on-line les brinda. Las respuestas de los usuarios de LaVanguardia.com muestran que, aunque lean ambas ediciones con asiduidad, no las consideran radicalmente distintas entre sí: no enfatizan ninguno de los rasgos distintivos de la edición on-line, tampoco le niegan a esta los valores de objetividad, confianza y actualidad que para ellos caracterizan a la edición impresa y, por extensión, a su marca periodística.

Podemos concluir, en consecuencia, que la complementariedad entre soportes no es el motivo principal de que estos lectores lean las dos ediciones de una misma cabecera. Ciertamente, las características específicas de cada soporte hacen que su uso pueda ser complementario, es decir, diverso. Pero la percepción que los lectores estudiados demuestran tener de ambas ediciones es unitaria. Mientras los usos de cada soporte no pueden sino divergir, los principales objetivos de los lectores cuando acceden a las dos ediciones de su cabecera de referencia convergen: con el uso simultáneo de 
ambos soportes lo que pretenden es seguir leyendo los contenidos de una marca periodística que identifican con los valores de actualidad, objetividad y confiabilidad. El soporte on-line amplía las posibilidades de acceso a esos contenidos. El sentido unitario de ambas ediciones es lo que ha movido a los lectores de la edición impresa a adoptar el uso del soporte on-line para realizar hoy ese tipo de consumo en ambos soportes. Por ende, a medida que Internet se vaya generalizando aún más, es muy probable que los numerosos lectores que hoy hacen una lectura exclusiva de la edición impresa vayan incorporándose a la edición on-line, siempre que puedan reconocer en ella los mismos valores que, a su juicio, encarna la marca periodística que han escogido como de referencia. Por ello, si una cabecera quiere reforzar o ampliar la relación con sus lectores, deberá apoyarse en aquello que, a través de la edición impresa, ya le ha ayudado a establecer un vínculo con la mayoría de ellos.

Mientras perdure la incógnita de hacia dónde van a evolucionar cada una de las dimensiones del proceso global de convergencia en el que las empresas periodísticas se hayan inmersas, aquello que los usuarios esperan hoy de cada cabecera es algo que ni las empresas periodísticas tradicionales ni la investigación deben perder de vista. Sabe- mos ahora que los lectores no identifican cada una de las ediciones de prensa con las plataformas en las que las leen, sino con la cabecera que las edita; para ellos son un producto de su marca periodística y esperan que eso quede confirmado en los contenidos a los que ahora pueden acceder a través de distintos soportes. Esas motivaciones que mueven hoy a los lectores a un consumo en ambos soportes, quizá constituyan una clave desde la que estudiar el proceso convergente. Nuestro análisis nos lleva a afirmar la necesidad de ir más allá del estudio de aquellas gratificaciones que los usuarios asocian a cada uno de los soportes que usan. Conviene también profundizar en todas aquellas dimensiones de gratificación que esperan obtener tanto de los contenidos como de la cabecera en la que han depositado su confianza, ya sea en uno o en múltiples soportes. No en vano, la reconfiguración de las empresas periodísticas como conglomerados multimedia y multiplataforma, ha comenzado a traducirse en una idea: la marca es más importante que el soporte (Salaverría, 2010). En el proceso de multiplicación de plataformas hacia el que parece tender la convergencia mediática (Cabrera, 2010: 13), las marcas periodísticas tradicionales parten con la gran ventaja de tener un público ya fidelizado. Está en su mano no perderla. 


\section{Referencias}

Asociación para la investigación de medios de comunicación (AIMC). (2011). La Prensa: digital vs. Papel. Recuperado de: www.aimc.es/-La-PrensaDigital-vs-Papel-.html (1/10/2011).

Armentia, J. I.; Caminos, J. M.; Elexgaray, J.; Martin, F., \& Merchán, I. (2000). El diario digital: Análisis de los contenidos textuales, aspectos formales $y$ publicitarios. Barcelona: Bosch.

Cabrera, M. A. (coord.) (2010). Evolución tecnológica y cibermedios. Zamora: Comunicación social.

Canga, J.; Coca, C.; Martínez, E.; Cantalapiedra, M. J. Y Martínez, L. (2000). Diarios digitales. Apuntes sobre un nuevo medio. Bilbao: Servicio Editorial De La Universidad Del País Vasco.

Chy, H. I. \& Lasorsa, D. (2002). An Explorative Study on the Market Relation Between Online and Print Newspapers. The Journal of Media Economics, 15(2), 91-106.

Chyi, H.I., Yang, M.J., Lewis, S.C., \& Zheng, N. (2010). Use of and satisfaction with newspaper sites in the local market: Exploring differences between hybrid and online-only users. Journalism and Mass Communication Quarterly. 87(1), 62-83.

Chy, H. I. ; Huang, J. S (2011). Demystifying the demand relationship between online and print products under one newspaper brand: the case of Taiwan and the emergence of a universal pattern. Asian Journal of Communication. 21, 243-261.

De Waal, E.; Schönbach, K. \& Lauf, E. (2005). Online Newspapers: A Substitute or Complement for Print Newspapers and Other Information Channels? Communications, 30, 55-72.

Díaz-Noci, J. (2010). Medios de comunicación en Internet: algunas tendencias. El profesional de la información, 19, 6, 561-567.

Estudio General De Medios, datos de las oleadas realizadas por la AIMC entre los años 2008 y 2010.

Filistruchi, L. (2005). The impact of Internet on the Market for Daily Newspapers in Italy. European University Institute, Working paper, 12.
Flavián, C., \& Gurrea, R. (2007). Perceived substitutability between digital and physical channels: the case of newspapers. Online Information Review, 31 (6), 793-813.

Gentzkow, M. (2006). Valuing New Goods in a Model with Complementarity: Online Newspapers. American Economic Review, 97(3), 713-744.

George, L. (2006). The Internet and the Market for Daily Newspapers. The BE Journal of Economic Analysis \&Policy.

López, X. \& Pereira, J. (Coord.) (2010). Convergencia digital. reconfiguración de los medios de comunicación en España. Santiago de Compostela: Servicio Editorial Universidad de Santiago de Compostela.

Masip, P., Díaz Noci, J., Domingo, D., Micó, J. L., \& Salaverrí-A, R. (2010). Investigación internacional sobre ciberperiodismo: Hipertexto, interactividad, multimedia y convergencia. El Profesional De La Información, 19(6), 568-576.

Mcquail, D. (1997). Audience analysis. Londres: Sage Publications, Inc.

Meyen, M. (2004). Mediennutzung. Konstanz: UVK.

Picard, R.G. (2003). Cash cows or entrecote: Publishing companies and Disruptive technologies.

Trends in Communication, 11(2), 127-136.

Rice, R. E. (1984). The new media. Communication Research Technology. California: Sage Publications.

Ruggiero, T. E. (2000). Uses and gratifications theory in the 21 st century. Mass Comunication \& Society. 3(1), 3-37.

Salaverría, R. (2010). "Concepto de convergencia periodística", en López, X. \& Pereira, J. (Coord.) (2010). Convergencia digital. reconfiguración de los medios de comunicación en España. Santiago de Compostela: Servicio Editorial Universidad de Santiago de Compostela.

Smith, A. (2011). Migración digital. ¿Es este el futuro del periódico?. Telos: Cuadernos de comunicación e información, 86, 98-100.

Valdettaro, S. (2009). "Diarios: entre Internet, la desconfianza y los árboles muertos”, en Carlón, $\mathrm{M}$. 
\& Scolari, C.A. (2009). El fin de los medios masivos. El comienzo de un debate. Buenos Aires: La Crujía.

Van Der Wuurf, R. (2005). Impacts of the Internet on Newspapers in Europe. Gazette: the international journal for communication studies, 67(1), 107-120.

Van Der Wurff, R.; Lauf, E; Balcytiene, A; Fortunati, L.; Holmberg, S.L.; Paulussen, S. \& Salaverria, R. (2008). Online and print newspapers in Europe in 2003. Evolving towards complementarity, Communications, 33, 403-430.

Westlund, O. \& Färdigh, M. A. (2011). Displacing and Complementing Effects of News Sites on Newspapers 1998-2009. The International Journal on Media Management, 13, 177-194.
Cita de este artículo

LACASA MAS, I.; VICTORIA MAS, M. y FERNÁNDEZ DIEZ, M. (2012) Confianza en la marca periodística y adopción de la prensa on-line. Revista Icono14 [en línea] 20 de Enero de 2012, Año 10, Vol. 1, pp. 213-229. Recuperado (Fecha de acceso), de http://www.icono14.net

\section{NOTAS}

${ }^{\text {i }}$ El presente artículo surge de un plan de investigación sobre la audiencia de la prensa digital realizado en el marco del proyecto CSO2009-13713-C05-02.

${ }^{\text {ii }}$ Estos autores entienden el proceso convergente como un continuum, un marco de referencia en el que los mercados periodísticos, empresas de información y/o medios se encuentran en un proceso que tiene como meta la integración (Salaverría, 2010: 47). La mayoría de redacciones muestran aún mínima coordinación organizacional entre sí: el $42.9 \%$ de las principales empresas periodísticas españolas comparten el mismo espacio físico, pero las colaboraciones se reducen a los contenidos y la coordinación entre ambas es mínima (López et. al. 2010; 322).

iii Según el último estudio de la AIMC sobre la lectura de prensa en soporte impreso y digital que realizan los internautas españoles, los dispositivos de acceso a periódicos on-line más utilizados siguen siendo el ordenador portátil y el ordenador de sobremesa, con un 66,9\% y un 55,1\%, respectivamente (AIMC, 2011: 18; la encuesta fue realizada a los individuos procedentes del año móvil de la $2^{\text {a }}$ ola del EGM de 2010 que habían accedido a Internet en los 30 días anteriores). Ese mismo estudio muestra que el acceso a diarios digitales desde teléfonos móviles $(16,6 \%)$ y desde tabletas (4,5\%) ya es relevante (AIMC, 2011: 18). En este artículo nos limitaremos a analizar el uso que hacen los lectores de prensa de cada uno de los dos soportes, impreso y digital, independientemente del dispositivo que utilicen para leer la edición on-line. A nuestro entender, si un lector de prensa lee habitualmente la edición on-line de su cabecera, también es susceptible de acceder habitualmente a sus contenidos a través de distintos dispositivos móviles, sobre todo cuando estos últimos hayan alcanzado una mayor difusión.

${ }^{\text {iv }}$ La penetración de un medio, en este caso de la edición impresa o de la edición digital de estas cabeceras, es el porcentaje que representa su audiencia con respecto al total de la población. No incluimos datos de ElMundo.es porque, a pesar de ser el diario on-line de mayor difusión en España, no está asociado a la AIMC, y el EGM no recoge sus datos (Vid: http://www.aimc.es/Sitios-de-Internet.html). 
${ }^{v}$ Como puede apreciarse en el Cuadro 1 que resume la variación de lectores para cada edición de cada cabecera, salvo en el caso de El País, el número de lectores que combinan ambas versiones crece incluso más que lo que disminuye el volumen de lectores de sus ediciones impresas.

vi Todavía está por ver si todos los productos periodísticos que se publican en ambos soportes — papel y digital— bajo la marca periodística de una misma cabecera están llamados a sobrevivir. "En las versiones más optimistas, es posible una asociación entre el prestigio del diario papel y la flexibilidad de la edición electrónica que, junto a los otros medios, conformarían conglomerados mediáticos exitosos para los cuales el aporte del mercado del papel tendría una función sistémica" (Valdettaro, 2009).

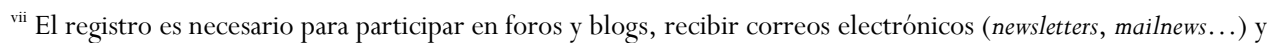
suscribirse a la edición impresa. La mayoría de nuestros encuestados estaban registrados para recibir la newsletter o porque eran suscriptores.

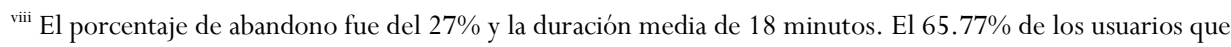
contestaron la encuesta fueron hombres y el 34.23\% mujeres. La distribución por franjas de edad fue la siguiente: $12.51 \%$ la de 26-35 años, $29.09 \%$ la de 36-45 años, $29.37 \%$ la de $46-55$ años y $17.55 \%$ la de 56-65 años. Para poder considerar a este grupo de lectores registrados una muestra representativa de todos los lectores de La Vanguardia que combinan la lectura de ambas versiones ha sido necesario equilibrar la muestra, a fin de que la distribución de porcentajes coincidiera con los porcentajes de las franjas de edad establecidas por el EGM. Según esos datos del EGM, los lectores pertenecientes a las franjas de edad de 35-44, 25-34 y 45-54 años son los más numerosos entre la audiencia de LaVanguardia.com que además lee la edición impresa. Cabe destacar que la distribución de edades que hemos obtenido en nuestra encuesta no difiere de la de los lectores que solo leen la edición digital ni — según el EGM — de la de los lectores de la prensa digital española en general. La única desviación respecto al EGM es una pequeño desplazamiento de dos años en los límites que marcan las horquillas de edad de los encuestados. No consideramos que esa diferencia suponga una alteración del valor de los resultados.

${ }^{\text {ix }}$ La perspectiva usuario-céntrica habla de consumidores que se rigen más por sus dinámicas que por las de los medios, de suerte que sus acciones no pretenden amoldarse a uno u otro medio, sino lograr unos objetivos, dar respuesta a ciertas necesidades u obtener determinadas gratificaciones mediante ellos (Ruggiero, 2000).

${ }^{\mathrm{x}}$ Este enfoque parte de la premisa de que los miembros de la audiencia eligen de forma consciente y motivada entre los diferentes canales mediáticos y entre los contenidos ofrecidos por los medios, en vistas a satisfacer unas necesidades propias que tienen origen social y psicológico (McQuail, 2000).

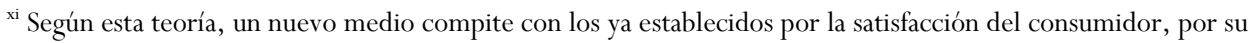
tiempo y por la inversión publicitaria; además, cuando el nuevo medio cumple algunas de las funciones de aquellos más antiguos, la consecuencia para estos últimos puede ser la exclusión, la sustitución o el desplazamiento, siendo esta última la más habitual (Dimmick et. al., 2004). Hay que tener en cuenta que la teoría del nicho se centra en las oportunidades de gratificación que ofrece el medio, es decir, en la dimensión de las gratificaciones que tiene que ver con las características de un medio y no con los atributos que le concede el consumidor individual a dicho medio, los cuales corresponderían a otras dos dimensiones de las gratificaciones: las percibidas y las obtenidas (Dimmick et. al., 2004).

${ }^{\text {xii }}$ Dentro del enfoque de usos y gratificaciones, el modelo de la expectativa-valor distingue entre las oportunidades de gratificación que un medio posee, y entre las gratificaciones buscadas por el usuario (expectativas) y las obtenidas con el uso de ese medio (McQuail 2000: 462). Este modelo postula que las expectativas y las evaluaciones influyen sobre la búsqueda de gratificaciones, la cual, a su vez, influye sobre el consumo de los medios, consumo que da lugar a una percepción de ciertas gratificaciones obtenidas, las cuales, entonces, retroalimentan — refuerzan o alteran — las percepciones que un individuo tiene acerca de aquellos atributos que asocia a alguna gratificación de un determinado medio (Palmgreen y Rayburn, 1985: 64). Las 
gratificaciones obtenidas no son creencias sino beneficios experimentados (cognitivos, afectivos o conductuales) en el consumo de los medios (Palmgreen y Rayburn, 1985: 66). El hecho de que resulte complejo definir y operacionalizar conceptos de una naturaleza tan cualitativa como son las expectativas o las gratificaciones ha suscitado no pocas críticas contra el enfoque de usos y gratificaciones, al que pertenece este modelo. A pesar de ello, son también numerosos los autores que hoy siguen defendiendo la validez este enfoque para el estudio del uso de los medios (Ruggiero 2000: 28; Meyen 2004).

${ }^{\text {xiii }}$ Ya en los estudios realizados acerca de la adopción del videotexto y de los primeros periódicos electrónicos las teorías sobre la lectura de prensa apuntaban como uno de los principales obstáculos para su adopción el hecho de que muchos de los lectores valorasen la lectura de prensa como un fin en sí mismo, y por ello, la mayor parte de veces no buscasen satisfacer unos objetivos tan concretos o específicos como puede permitir con mayor eficacia el soporte digital que el soporte tradicional (Rice et. al. 1984). Al mismo tiempo, estos autores afirman que los lectores que están motivados principalmente por las necesidades relacionadas con el ocio prefieren consumir un producto editado y con una forma y contenido bien determinados (Rice et. al. 1984).

${ }^{\text {xiv }}$ Para el presente artículo se han seleccionado solo algunas de las 40 preguntas que componían la encuesta completa. No pretendemos hacer una exposición global de los resultados. Queremos centrarnos en una cuestión muy concreta, tal como la hemos presentado más arriba en la introducción, la hipótesis y los objetivos.

${ }^{\mathrm{xv}}$ Su puntuación no supera por mucho el 3. En una puntuación del 1 al 5, el 3 corresponde a una opción que denota más bien indiferencia o indefinición. 